\title{
COVID-19 Mortality Following Mass Gatherings
}

\author{
Oren Miron M.A., Kun-Hsing Yu M.D., Ph.D., Rachel Wilf-Miron, M.D., M.P.H., Nadav \\ Davidovitch. M.D., M.P.H
}

Author affiliation: Department of Health Systems Management, Ben-Gurion University of the

Negev, Beer Sheva, Israel (Miron and Davidovitch). Department of Biomedical Informatics, Harvard Medical School, Boston, Massachusetts (Yu). Faculty of Medicine, Tel Aviv University, Tel Aviv, Israel (Wilf-Miron).

Corresponding author: Oren Miron, MA, Department of Health Systems Management, BenGurion University, 1 David Ben Gurion Blvd, 84105 ,Beer Sheva, Israel (orenmir@ post.bgu.ac.il; 972-8-6472448 ).

\begin{abstract}
:
We examined Coronavirus Disease-2019 (COVID-19) mortality following 5 mass gatherings at outdoor rallies in the United States, during August 2020. We found that COVID-19 mortality started increasing 19-24 days after the mass gathering. In a 50-mile radius there was a 2.1-fold increase in COVID-19 mortality, and in a 51-100 miles radius there was a 1.4-fold increase. Our results suggest that precautions should be taken in mass gatherings and in at least a 50-mile radius, in order to limit COVID-19 mortality.
\end{abstract}




\section{Introduction:}

Mass gatherings have been linked to Coronavirus Disease-19 (COVID-19) deaths at the start of the pandemic, which led governments to temporarily limit most mass gatherings. ${ }^{1}$ This makes it difficult to determine the effect of mass gatherings on COVID-19 mortality in the later stage of the pandemic, which has more testing and treatments. ${ }^{2}$

If mass gatherings are followed by increased mortality, there is a need to estimate the radius of this effect in order to increase precautions in that region.

\section{Methods:}

Five mass gatherings occurred in August 2020 at outdoor rallies, in the states of Minnesota (August $17^{\text {th }}$ ), Wisconsin (August $17^{\text {th }}$ ), Arizona (August $18^{\text {th }}$ ), Pennsylvania (August 20 ${ }^{\text {th }}$ ), and New-Hampshire (August $28^{\text {th }}$ ). We retrospectively extracted the daily COVID-19 mortality of each county in those states in the 30 days following each mass gathering. ${ }^{3}$

We aggregated the counties based on distance from their center coordinates to the coordinates of the mass gathering in their state, with groups of 0-50 miles, 51-100 miles, above 100 miles. ${ }^{4} \mathrm{We}$ also extracted the mortality in all the states that did not have those mass gatherings in August 2020, and we defined their control mass gathering date as the median date of the August mass gatherings (August $18^{\text {th }}$ ). 
We calculated the mortality rate per 100,000 capita with a 7-day moving-average, and examined its trend using the JoinPoint Regression Program (National Cancer Institute), and a 2-tailed statistical test with a significance level of 0.05 . Lastly, we examined the rate and $95 \%$ confidence interval (CI) of the incidence when the gatherings are expected to have effects on deaths (20 days post-gathering $)^{5}$, and compared it with the rate at 30 days post-gathering.

\section{Results:}

At 0-50 miles from the mass gathering, the COVID-19 death rate decreased from gathering day to day 24 (-1.6\% daily-change, $95 \%$ confidence interval $-2.4 \%$ to $-0.9 \%)$, followed by an increase until day 30 (7.8\% daily-change, $95 \%$ confidence interval $1.4 \%$ to $14.6 \%$ ). At day 20 the rate was $0.07 / 100,000$ capita (95\% confidence interval, 0.04 to 0.1 ), and at day 30 it increased to $0.15 / 100,000$ capita (95\% confidence interval, 0.11 to $0.2,2.1$-fold increase; Figure 1). 


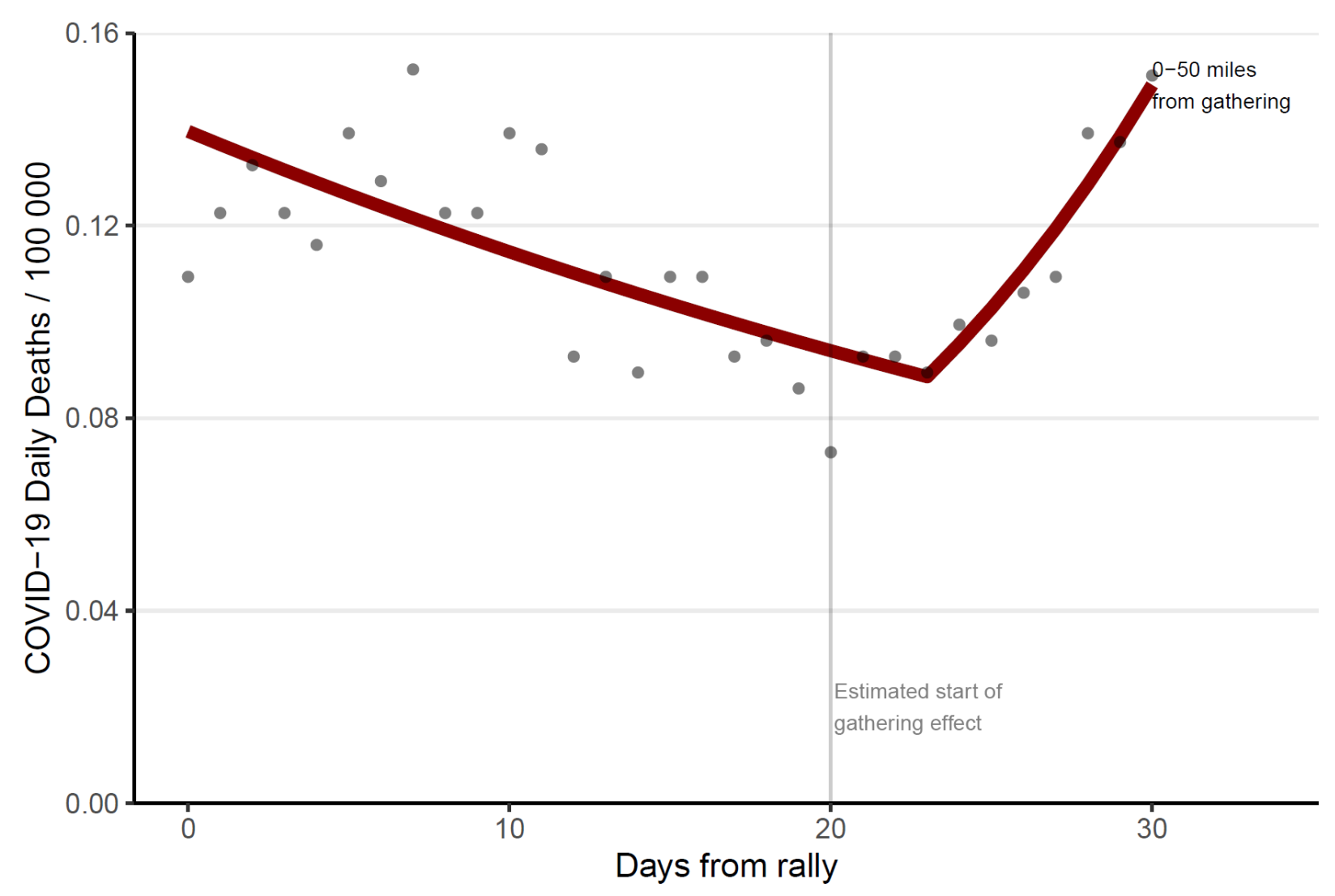

Figure 1: COVID-19 mortality by days in a 50 miles radius from mass gathering

Caption: Y indicates Coronavirus Disease-19 deaths per 100000 capita, and X indicates days from mass gathering. Dots indicate the observed data points from counties in the state of the mass gathering who were 50 miles or less from the gathering location. The line indicates the trend based on JoinPoint regression.

At 51-100 miles, the COVID-19 death rate decreased from gathering day to day 19 (-1.9\% dailychange, $95 \%$ confidence interval $-2.3 \%$ to $-1.5 \%)$, followed by an increase until day 30 (3.9\% daily-change, $95 \%$ confidence interval $2.9 \%$ to $4.8 \%$ ). At day 20 the rate was $0.1 / 100,000$ capita ( $95 \%$ confidence interval, 0.08 to 0.12 ), and at day 30 it increased to $0.15 / 100,000$ capita ( $95 \%$ confidence interval, 0.12 to 0.17 , 1.4-fold increase; Figure 2). 


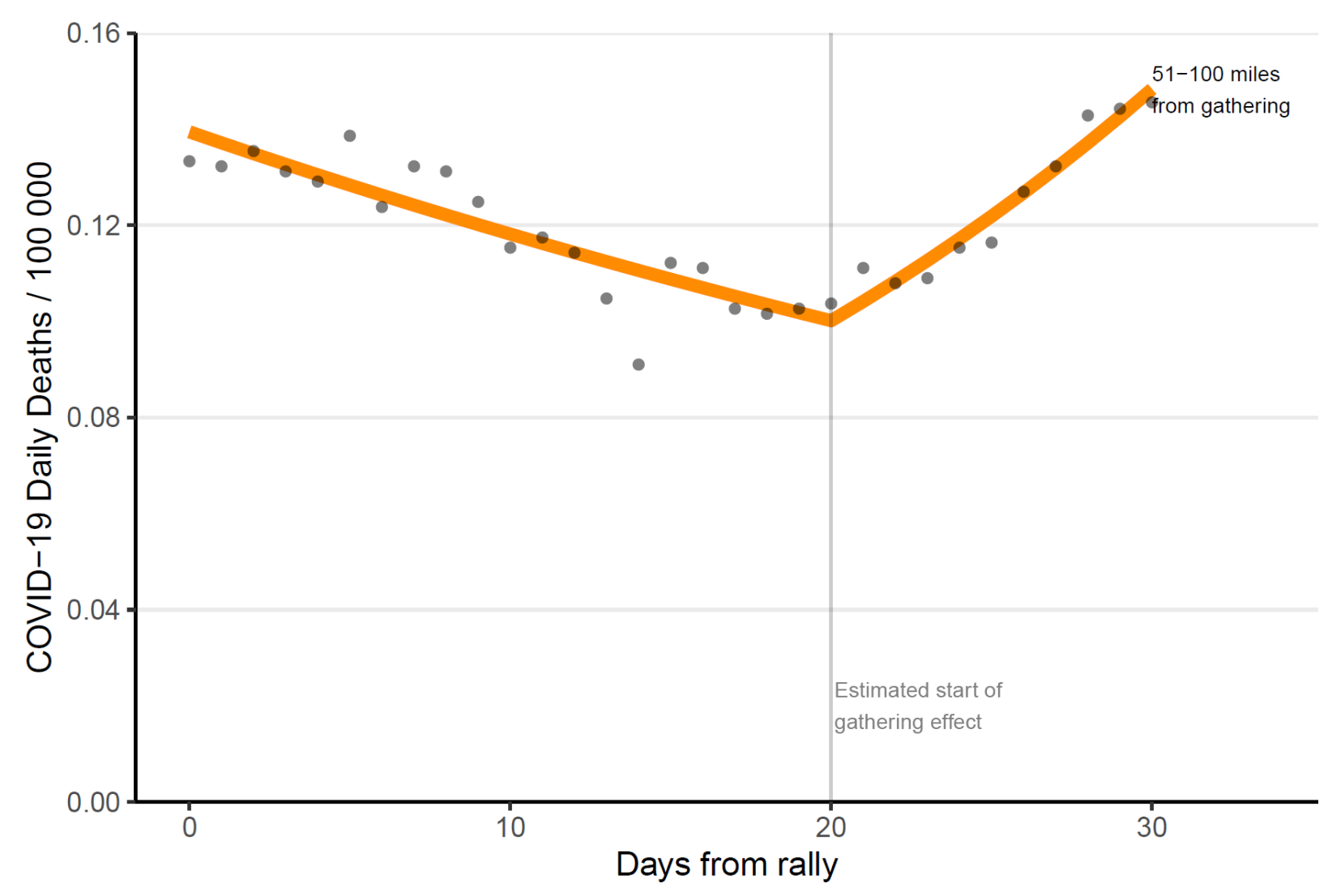

Figure 2: COVID-19 mortality by days in a 51-100 miles radius from mass gathering

Caption: Y indicates Coronavirus Disease-19 deaths per 100000 capita, and $\mathrm{X}$ indicates days from mass gathering. Dots indicate the observed data points from counties in the state of the mass gathering who were between 50-100 miles from the gathering location. The line indicates the trend based on JoinPoint regression. 
At over 100 miles, the COVID-19 death rate decreased from gathering day to day $25(-2.7 \%$ daily-change, $95 \%$ confidence interval $-3.3 \%$ to $-2.1 \%$ ), followed by a daily change of $7 \%$ (95\% confidence interval $-0.4 \%$ to $15 \%)$. At day 20 the rate was $0.18 / 100,000$ capita $(95 \%$ confidence interval, 0.15 to 0.2 ), and at day 30 it increased to $0.22 / 100,000$ capita (95\% confidence interval, 0.2 to $0.25,1.2$-fold increase; Figure 3 ).

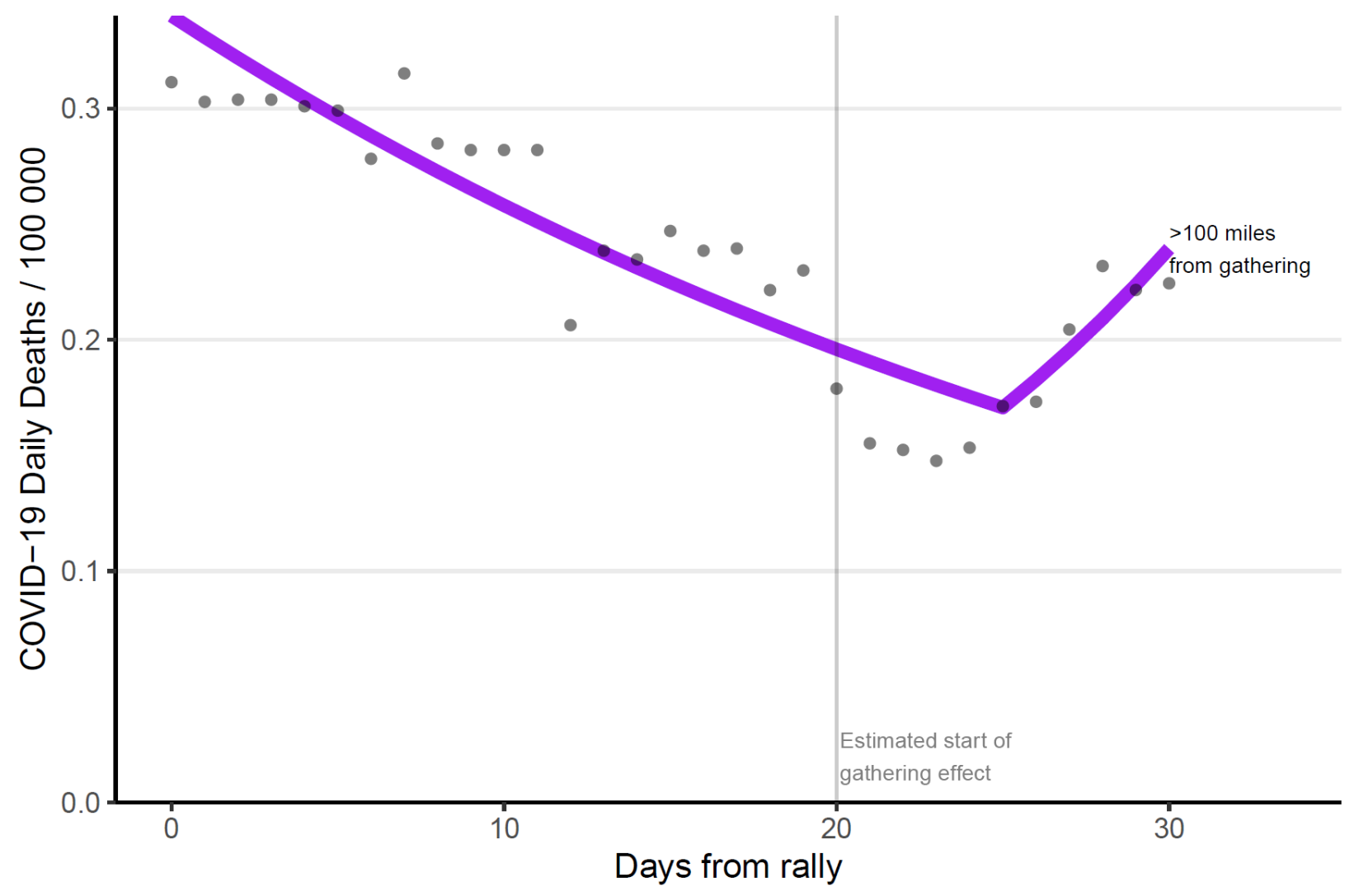

Figure 3: COVID-19 mortality by days in 100 miles or more radius from mass gathering

Caption: Y indicates Coronavirus Disease-19 deaths per 100000 capita, and X indicates days from mass gathering. Dots indicate the observed data points from counties in the state of the mass gathering who were over 100 miles from the gathering location. The line indicates the trend based on JoinPoint regression. 
In states without the mass gatherings, the COVID-19 death rate decreased from control gathering day to day 20 ( $-1.1 \%$ daily-change, $95 \%$ confidence interval $-1.4 \%$ to $-0.8 \%)$, followed by an increase until day 30 (1.1\% daily-change, $95 \%$ confidence interval $0.2 \%$ to $2.1 \%$ ). At day 20 the rate was $0.27 / 100,000$ capita (95\% confidence interval, 0.26 to 0.27 ), and at day 30 it increased to $0.3 / 100,000$ capita (95\% confidence interval, 0.29 to $0.31,1.1$-fold increase; Figure 4 ).

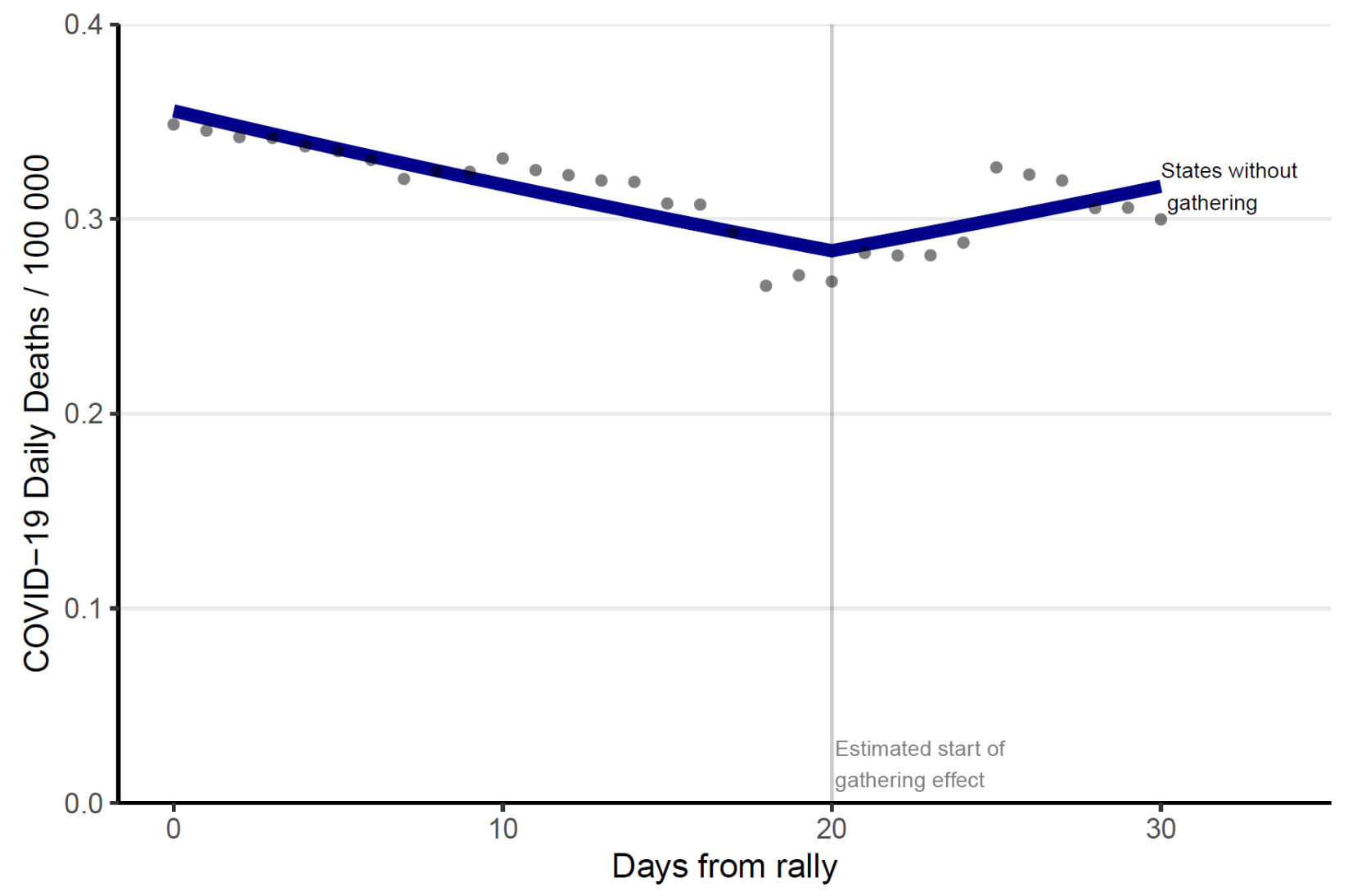

Figure 4: COVID-19 mortality by days from control date in states without mass gatherings

Caption: Y indicates Coronavirus Disease-19 deaths per 100000 capita, and X indicates days from mass gathering control date (median date of the 5 mass gatherings). Dots indicate the observed data points from counties in states who did not have any of the 5 mass gatherings that were analyzed. The line indicates the trend based on JoinPoint regression. 


\section{Discussion:}

Our analysis showed an increase in COVID-19 mortality following mass gatherings, with the highest increase at 0-50 miles from the mass gathering (2.1-fold), followed by 51-100 miles (1.4fold), 100+ miles (1.2-fold), and in states without the mass gatherings (1.1-fold).

The mortality increase in states without mass gatherings could stem from unrelated factors, ${ }^{6,7}$ or relate to out-of-state participants in the mass gatherings, such as the Georgia resident who died of COVID-19 after attending an Oklahoma mass gathering without a mask or distancing. ${ }^{8}$ States could promote masks and distancing in mass gatherings, and in at least a 50-mile radius from the mass gathering, which may limit COVID-19 mortality.

\section{ARTICLE INFORMATION}

Author Contributions: Oren Miron had full access to all of the data in the study and takes responsibility for the integrity of the data and the accuracy of the data analysis.

Concept and design: All authors.

Acquisition, analysis, or interpretation of data: All authors.

Drafting of the manuscript: Oren Miron. 
Critical revision of the manuscript for important intellectual content: Yu, Wilf-Miron, and Davidovitch.

Statistical analysis: Oren Miron and Yu.

Study supervision: Davidovitch.

Conflict of Interest Disclosures: None reported.

Funding/Support: No external funding

Data availability statement: publicly available at

https://github.com/CSSEGISandData/COVID-19

\section{References:}

1. Wiersinga WJ, Rhodes A, Cheng AC, Peacock SJ, Prescott HC. Pathophysiology, transmission, diagnosis, and treatment of coronavirus disease 2019 (COVID-19): a review. Jama. 2020;324(8):782-793.

2. Gostin LO, Wiley LF. Governmental public health powers during the COVID-19 pandemic: stay-at-home orders, business closures, and travel restrictions. Jama. 2020;323(21):21372138. 
3. Presidential candidate campaign travel, 2020. Ballotpedia. Accessed September 25, 2020. https://ballotpedia.org/Presidential_candidate_campaign_travel,_2020

4. Dong E, Du H, Gardner L. An interactive web-based dashboard to track COVID-19 in real time. The Lancet infectious diseases. 2020;20(5):533-534.

5. Auger KA, Shah SS, Richardson T, et al. Association between statewide school closure and COVID-19 incidence and mortality in the US. JAMA. Published online 2020.

6. Stein-Zamir C, Abramson N, Shoob H, et al. A large COVID-19 outbreak in a high school 10 days after schools' reopening, Israel, May 2020. Eurosurveillance. 2020;25(29):2001352.

7. Im Kampe EO, Lehfeld A-S, Buda S, Buchholz U, Haas W. Surveillance of COVID-19 school outbreaks, Germany, March to August 2020. Eurosurveillance. 2020;25(38):2001645.

8. Wang X, Pasco RF, Du Z, et al. Impact of Social Distancing Measures on Coronavirus Disease Healthcare Demand, Central Texas, USA. Emerg Infect Dis. 2020;26. 